\title{
Expression of macrophage inhibitory cytokine-1 in early gastric cancer cases treated using endoscopic mucosal resection and the correlation with prognosis
}

\author{
NA ZHANG and BO LU \\ Xuzhou Hospital Affiliated to Medical College of Southeast University, Xuzhou, Jiangsu 221009, P.R. China
}

Received May 13, 2016; Accepted February 2, 2017

DOI: $10.3892 / \mathrm{ol} .2017 .6376$

\begin{abstract}
The expression of macrophage inhibitory cytokine-1 (MIC-1) of early gastric cancer treated using endoscopic mucosal resection (EMR) and its correlation with prognosis were investigated. One hundred and forty-seven patients with early gastric cancer (less than $20 \mathrm{~mm}$ in diameter) were recruited and assigned into an observation group (75 cases) or a control group (72 cases), according to treatment. The control group was treated with a radical resection by the conventional laparotomy approach and the observation group was treated by EMR. Differences in operative outcomes and prognosis were compared between the two groups. Complete lesion resection rates of the two groups were $100 \%$. The amount of bleeding, operation time and postoperative complication rates in the observation group were each significantly lower than those in the control group $(\mathrm{P}<0.05)$. The serum MIC-1 levels in the two groups were reduced, with the observation group being significantly lower than that of the control group $(\mathrm{P}<0.05)$. The progression-free survival was significantly prolonged and the recurrence rate was significantly lower in the observation group compared to the control group, with the difference being statistically significant $(\mathrm{P}<0.05)$. In conclusion, EMR treatment of early gastric cancer was observed to be safe and effective in this study and can reduce the expression level of serum MIC-1 after the operation. EMR can also increase the survival period and reduce the recurrence rate, and $\mathrm{MIC}-1$ can be used as an important index to predict the prognosis of the disease.
\end{abstract}

\section{Introduction}

Early gastric cancer refers to gastric cancer confined to the mucosa or submucosa, regardless of local lymph node

Correspondence to: Dr Bo Lu, Xuzhou Hospital Affiliated to Medical College of Southeast University, 199 South Road, Xuzhou, Jiangsu 221009, P.R. China

E-mail: lubo_1973@163.com

Key words: endoscopic mucosal resection, early gastric cancer, macrophage inhibitory cytokine-1, progression-free survival, recurrence rate metastasis. The postoperative survival rate after 5 years of early gastric cancer is $>90 \%$ (1). With the use of confocal endoscopy, narrow band imaging, and magnification chromoendoscopy, an increasing number of gastric cancers are found in the early stage (2).

The preferred treatment for early gastric cancer is traditional radical surgery with lymph node dissection, but the rate of postoperative complications is up to $43 \%$. Radical resection alters the normal anatomical structure and can cause postoperative dysphagia, regurgitation, loss of appetite, weight loss and other symptoms, severely reducing the quality of life of the patient (3). Endoscopic mucosal resection (EMR) can simultaneously complete the diagnosis and treatment of gastric cancer in one operation. With its resultant reduced risk of trauma, rapid recovery and high complete resection rate, its application gradually increases in the clinical setting (4). Macrophage inhibitory cytokine (MIC)-1 was recently found to have a close relationship with the occurrence of gastric cancer, and its high expression often indicates a poor longterm prognosis (5).

The findings from research on EMR treatment of early gastric cancer at our center is summarized as follows.

\section{Patients and methods}

Patient data. One hundred forty-seven cases of gastric cancer were diagnosed via biopsy with pathological confirmation at the Xuzhou Hospital Affiliated to Medical College of Southeast University (Jiangsu, China), from January 2010 to January 2013, and were selected continuously for participation in the study. Inclusion criteria were: i) Age $\geq 18$ years; ii) the tumor diameter was $<20 \mathrm{~mm}$ and not associated with erosion or ulcer; and iii) infiltration depth and scope of the mucosa had enough safety margins for resection. Exclusion criteria were: i) Patients with coagulopathy; ii) patients with other malignant tumors; iii) patients with comorbidities of the heart, liver and/or kidney; and iv) patients not willing to participate in the study.

This study was approved by the Ethics Committee of Xuzhou Hospital Affiliated to Medical College of Southeast University. Signed written informed consents were obtained from all participants before the study. Patients were informed of the detailed surgical conditions and the possible risks of adverse events and complications before surgery and informed written consent of the patients or their families was obtained. 
Table I. Comparison of blood loss, operative time and postoperative complication rate.

Blood loss in Operative Blood loss after

Complication

Groups Cases operation (ml) time (min) operation (\%)

Infection (\%) Perforation (\%) Others (\%) incidence (\%)

\begin{tabular}{lcccccccc}
\hline Observation & 75 & $151.2 \pm 38.4$ & $58.3 \pm 14.5$ & $3(4.0)$ & $1(1.3)$ & $1(1.3)$ & $1(1.3)$ & $6(8.0)$ \\
Control & 72 & $258.3 \pm 41.2$ & $86.4 \pm 23.2$ & $6(8.3)$ & $2(2.8)$ & $3(4.2)$ & $3(4.2)$ & $14(19.4)$ \\
$\mathrm{t}\left(\chi^{2}\right)$ test & & 6.495 & 7.147 & & & & & 4.093 \\
P-value & & 0.033 & 0.026 & & & & & 0.043 \\
\hline
\end{tabular}

The patients were then divided into the observation group ( 75 cases) and the control group (72 cases), according to the chosen treatment method. There were 41 male and 34 female cases in the observation group. Ages ranged from 37 to 75 , with an average of $51.9 \pm 6.2$ years. There were 46 cases with a differentiated type of morphology and 29 cases with an undifferentiated type. Thirty-five cases were intra-mucosal cancer, whereas 40 cases were submucosal cancer. The control group consisted of 44 male and 28 female cases. Ages ranged from 35 to 76, with an average of 52.1 \pm 7.2 years. There were 40 cases with a differentiated type of morphology and 32 cases with an undifferentiated type. Thirty-one cases were intramucosal cancer, whereas 41 cases were submucosal cancer. The distribution of these general factors among patients between the two groups showed no statistical differences $(\mathrm{P}>0.05)$.

Research methods. The two groups of patients were treated using the same surgical and healthcare team, according to the standard medical process. The control group underwent radical resection with conventional laparotomy as follows: Administration of general anesthesia, access by laparotomy into the lateral rectus side, cutting off most of the gastric tissue along the transverse colon and freeing the greater omentum behind, and then reconstructing the digestive tract, with placement of a conventional drainage tube.

Patients of the observation group were treated using EMR, as follows: Endoscopy was performed for observation of the lesion size and shape, and the depth of invasion. After the lesion was determined, the junction between the lesion and normal mucosa was stained with indigo carmine, and electric coagulation markers were made every $0.5 \mathrm{~cm}$, starting at $0.5 \mathrm{~cm}$ away from the junction. Using mixed liquid containing glycerol fructose and methylene blue to inject at multiple points, each point was injected with $\sim 2 \mathrm{ml}$. The injection was repeated until the lesion became notably elevated relative to the normal mucosa. After the lesion was ridged upwards, the disposable snare was stretched into the lesion from the biopsy channel until the lesion was completely set into the snare. Once the snare was incised into the muscularis propria, it was activated and the lesion was resected. After the excision, electrocautery was used for coagulation and hemostasis of blood vessels. Lesion tissue was fixed with neutral formalin and sent for pathological examination. Following the procedure, we carried out routine postoperative measures to ensure infection prevention, fluid infusion and hemostasis. In cases where perforation occurred during the surgery requiring suturing of the wound, delayed postoperative feeding time was necessary.
Observation index. A follow-up period was used to determine and compare progression-free survival and recurrence rates between the two groups. The deadline for follow-up of patients was January 2016 and the average follow-up period was 58.5 months. The amount of bleeding, operation time and postoperative complication rate were also compared between the two groups. The serum MIC-1 levels in the preoperative period and 3 months postoperative were compared. Detection of MIC-1 was as follows: Collection of $\sim 5 \mathrm{ml}$ of early-morning fasting peripheral venous blood was centrifuged at $3,000 \mathrm{rpm}$ for $20 \mathrm{~min}$, and then stored at $-20^{\circ} \mathrm{C}$. Concentration of MIC-1 was then tested, using the standard double antibody sandwich enzyme-linked immunosorbent assay (DAS-ELISA) detection methods with strict adherence to protocol (DAS-ELISA kit was purchased from the Beijing Zhongshan Jinqiao Biological Co., Ltd., Beijing, China). A routine abdominal CT scan was performed after the surgery at one, six, and then at six-month intervals with enhanced scanning used as necessary to evaluate any progression of the tumor.

Statistical analysis. SPSS 22.0 (Chicago, IL, USA) was used to process the data and for statistical analysis. The data were expressed as standard deviation (mean $\pm \mathrm{SD}$ ) and enumeration data were expressed as cases or (\%). Comparisons between groups were made using independent sample t-tests, and paired t-tests were used within groups for repeated measures. Further analyses of factors between groups were done using revised Chi-square tests and log-rank Chi-square tests. Kaplan-Meier survival analysis was used for assessment of progression-free survival. For all tests, $\mathrm{P}<0.05$ was considered statistically significant.

\section{Results}

Comparison of blood loss, operative time and postoperative complication rate. The lesion total resection rate was $100 \%$ and the resection margins were negative for both the observation and the control groups. The amount of bleeding, operation time and postoperative complication rate in the observation group were significantly lower than those in the control group (Table I), with the difference being statistically significant $(\mathrm{P}<0.05)$.

Comparison of serum MIC-1 levels between the pre-operative period and 3 months post-operative. Comparison of preoperative serum MIC-1 levels between the two groups showed no statistical difference $(\mathrm{P}>0.05)$. Postoperative serum MIC-1 levels in the two groups decreased compared 
Table II. Comparison of serum MIC-1 levels between the pre-operation and 3 months post-operation $(\mathrm{pg} / \mathrm{ml})$.

\begin{tabular}{lccrr}
\hline Groups & Pre-operation & Post-operation & t-test & P-value \\
\hline Observation & $237.5 \pm 42.3$ & $75.6 \pm 14.5$ & 13.630 & $<0.001$ \\
Control & $226.8 \pm 57.8$ & $124.3 \pm 38.2$ & 6.134 & 0.034 \\
t-test & 0.821 & 6.963 & & \\
P-value & 0.234 & 0.028 & & \\
\hline
\end{tabular}

MIC-1, macrophage inhibitory cytokine-1.

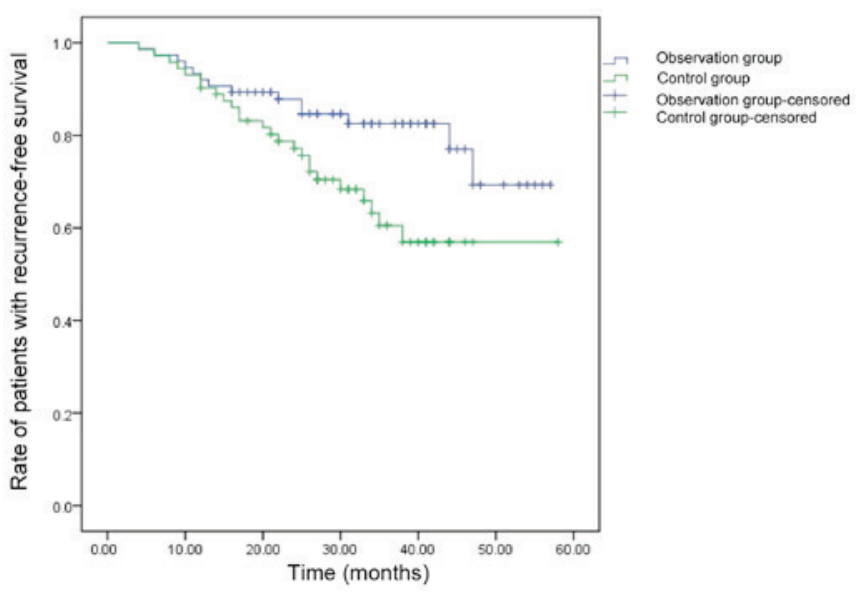

Figure 1. Survival rate of recurrence-free patients in the two groups.

with the preoperative levels, and the serum MIC-1 levels in the observation group were significantly lower than those in the control group (Table II), with the difference statistically significant $(\mathrm{P}<0.05)$.

Comparison of progression-free survival and recurrence in follow-up. The progression-free survival was more prolonged and the recurrence rate was lower in the observation group compared to the control group (Table III and Fig. 1), with the differences both being statistically significant $(\mathrm{P}<0.05)$.

\section{Discussion}

Gastrointestinal cancer, especially gastric cancer, has high incidence and mortality rates in China. Primary reasons for the high rates are insufficient patient screening and public health surveillance, limiting the capacity for early diagnosis.
Most gastric cancers are in middle or advanced stages when diagnosed clinically (6). Early gastric cancers are confined to the mucosa and submucosa tissue, making the risk of lymph node metastasis relatively low. Endoscopic examination can enable early detection, diagnosis and treatment of gastric cancer before it reaches advanced stages. Minimally invasive endoscopic treatment for early gastric cancer, compared with conventional surgery, also has the advantages of reduced risk of trauma, less postoperative complications, less intensive operative procedures, and can effectively improve the life quality of patients. While clear clinical efficacy is evident for treatment of early gastric cancer by radical resection, the disadvantages are an increased risk of trauma, slow postoperative recovery, delayed transfer time, prolonged hospitalization and greater healthcare costs, resulting in greater potential for physical and mental health issues and economic burden for the patient (7). Therefore, EMR has become one of the most effective methods for the diagnosis and treatment of early gastric cancer.

Currently, there are four kinds of EMR: Peeling off biopsy resection, standard EMR, cap-EMR and EMR with ligation. Standard EMR is applied most often, the diseased tissue is cut off after being lifted completely with a snare (8). It can be difficult to cut the whole lesion using EMR when the diameter exceeds $20 \mathrm{~mm}$. Therefore, it can become necessary to use endoscopic piece mucosal resection (EPMR); however, studies show (9) postoperative recurrence rate following EPMR is relatively higher than EMR. Broken specimens from EPMR are also difficult to use for accurate determination of surgical margins and depth of invasion.

Through this study, the complete resection rate was $100 \%$ for both the observation and the control groups. The amount of bleeding, operation time and postoperative complication rate in the observation group were significantly lower than those in the control group, which suggests that EMR has better safety and efficacy. The serum MIC-1 levels of the two groups of patients were both reduced, with the observation group significantly lower than the control group.

MIC-1, as a member of the TGF- $\beta$ family, is widely involved in cell apoptosis, metastasis and invasion, and other biological mechanisms (10). In normal serum, mature MIC-1 protein have low levels of expression, whereas in pathological conditions, such as malignancy, inflammation and acute injury, MIC-1 expression levels can be significantly increased, and the duration of its expression significantly longer (11). Kim et al (12) found that the MIC-1 gene was overexpressed in gastric cancer tissue, and its role varied in different stages of the development of gastric cancer.

Table III. Comparison of progression-free survival and recurrence in follow-up.

\begin{tabular}{|c|c|c|c|c|c|}
\hline Groups & Cases & $\begin{array}{l}\text { Progression-free } \\
\text { survival (months) }\end{array}$ & $\begin{array}{l}\text { Recurrence in } \\
\text { follow-up of } 1 \text { year }\end{array}$ & $\begin{array}{c}\text { Recurrence in } \\
\text { follow-up of } 3 \text { years }\end{array}$ & $\begin{array}{l}\text { Recurrence in } \\
\text { follow-up of } 5 \text { years }\end{array}$ \\
\hline Observation & 75 & 46.8 & $2(2.7)$ & $4(5.3)$ & $6(8.0)$ \\
\hline Control & 72 & 37.9 & $3(4.2)$ & $11(15.3)$ & $14(19.4)$ \\
\hline$\chi^{2}$ & & 15.632 & 0.002 & 3.965 & 4.093 \\
\hline P-value & & $<0.001$ & 0.963 & 0.046 & 0.043 \\
\hline
\end{tabular}


Lee et al (13) found that the expression of MIC-1 mRNA increased significantly with chronic inflammatory reaction, precancerous lesions and gastric cancer development, as well as invasion and metastasis of gastric carcinoma, indicating that MIC-1 expression accompanies tumor progression, and is closely related with tumor growth, invasion and metastasis. MIC-1 mRNA expression of gastric cancer with lymph node metastasis or distant metastasis was significantly higher than that without metastasis, indicating that expression is related to tumor metastasis. MIC-1 acts as a tumor suppressor in early gastric cancer by several mechanisms, including activating the P53 pathway (14), inhibiting COX-2 expression (15), inducing caspase-8 pathway (16), and activating the phosphatidylinositol 3-kinase/serine/threonine protein kinase and glycogen synthase $3 \beta$ signaling pathway (17). Conversely, it is also involved in advanced gastric cancer with promoting effects by inhibiting catenin $\delta 1$ gene expression (18), upregulating uPA system to enhance invasiveness of gastric cancer cells (19) and inducing the overexpression of ErbB2 receptor tyrosine kinase in gastric cancer cells (12). These and other mechanisms illustrate its role as an indicator of prognosis for early gastric cancer cases. Therefore, MIC-1 expression may be a manifestation of poor prognosis.

Our study showed that treatment of early gastric cancer with EMR resulted in significantly prolonged progression-free survival and significantly reduced rates of operative complications and disease recurrences compared to traditional surgery, suggesting that EMR in the treatment of early gastric cancer is both safe and effective. EMR can also reduce the postoperative expression of serum MIC-1, an important index for predicting the prognosis of the disease.

\section{References}

1. Lee JY, Cho KB, Kim ES, Park KS, Lee YJ, Lee YS, Jang BK, Chung WJ and Hwang JS: Risk factors for local recurrence after en bloc endoscopic submucosal dissection for early gastric cancer. World J Gastrointest Endosc 8: 330-337, 2016.

2. Pirogov SS, Sokolov VV, Karpova ES, Pavlov PV, Volchenko NN and Kaprin AD: Early gastric cancer and precancerous conditions diagnostics with confocal laser endomicroscopy. Eksp Klin Gastroenterol 3: 18-24, 2014 (In Russian).

3. Jianhui C, Yulong H, Chuangqi C, Kaiming W, Xinhua Z and Shirong C: Comparison of clinicopathological features of gastric carcinoma, between 2 times periods, at a single institute in China. J Cancer Res Ther 11: 874-881, 2015.

4. Hoteya S, Iizuka T, Kikuchi D, Ogawa O, Mitani T, Matsui A, Furuhata T, Yamashita S, Yamada A and Kaise M: Clinicopathological outcomes of patients with early gastric cancer after non-curative endoscopic submucosal dissection. Digestion 93: 53-58, 2016.

5. Lu Z, Yang L, Yu J, Lu M, Zhang X, Li J, Zhou J, Wang X, Gong J, Gao J, et al: Change of body weight and macrophage inhibitory cytokine-1 during chemotherapy in advanced gastric cancer: what is their clinical significance? PLoS One 9: e88553, 2014.
6. Pei H, Pu H, Dai M, Bai Y, Chang S, Wang Z, Cheng N, Li H, $\mathrm{Li} \mathrm{J}, \mathrm{Hu} \mathrm{X}$, et al: Disease burden of gastric cancer in Jinchang cohort. Zhonghua Liu Xing Bing Xue Za Zhi 37: 316-320, 2016 (In Chinese).

7. Sun PD, Cao H, Zhu JM and Fang XD: Value of radical dissection with vagus nerve preservation for proximal gastric cancer. Zhonghua Wei Chang Wai Ke Za Zhi 14: 117-119, 2011 (In Chinese).

8. Meng FS, Zhang ZH, Wang YM, Lu L, Zhu JZ and Ji F: Comparison of endoscopic resection and gastrectomy for the treatment of early gastric cancer: a meta-analysis. Surg Endosc 10: 125-126, 2015

9. Zhang YM, Boerwinkel DF, Qin X, He S, Xue L, Weusten BL, Dawsey SM, Fleischer DE, Dou LZ, Liu Y, et al: A randomized trial comparing multiband mucosectomy and cap-assisted endoscopic resection for endoscopic piecemeal resection of early squamous neoplasia of the esophagus. Endoscopy 48: 330-338, 2016.

10. Skipworth RJ, Deans DA, Tan BH, Sangster K, PatersonBrown S, Brown DA, Hunter M, Breit SN, Ross JA and Fearon KC: Plasma MIC-1 correlates with systemic inflammation but is not an independent determinant of nutritional status or survival in oesophago-gastric cancer. Br J Cancer 102: 665-672, 2010.

11. Baek KE, Yoon SR, Kim JT, Kim KS, Kang SH, Yang Y, Lim JS, Choi I, Nam MS, Yoon M, et al: Upregulation and secretion of macrophage inhibitory cytokine-1 (MIC-1) in gastric cancers. Clin Chim Acta 401: 128-133, 2009.

12. Kim KK, Lee JJ, Yang Y, You KH and Lee JH: Macrophage inhibitory cytokine-1 activates AKT and ERK-1/2 via the transactivation of ErbB2 in human breast and gastric cancer cells. Carcinogenesis 29: 704-712, 2008.

13. Lee DH, Yang Y, Lee SJ, Kim KY, Koo TH, Shin SM, Song KS, Lee YH, Kim YJ, Lee JJ, et al: Macrophage inhibitory cytokine-1 induces the invasiveness of gastric cancer cells by up-regulating the urokinase-type plasminogen activator system. Cancer Res 63: 4648-4655, 2003.

14. Yang H, Filipovic Z, Brown D, Breit SN and Vassilev LT: Macrophage inhibitory cytokine-1: a novel biomarker for p53 pathway activation. Mol Cancer Ther 2: 1023-1029, 2003.

15. Pang RP, Zhou JG, Zeng ZR, Li XY, Chen W, Chen MH and Hu PJ: Celecoxib induces apoptosis in COX-2 deficient human gastric cancer cells through Akt/GSK3beta/NAG-1 pathway. Cancer Lett 251: 268-277, 2007.

16. Huang Y, He Q, Hillman MJ, Rong R and Sheikh MS: Sulindac sulfide-induced apoptosis involves death receptor 5 and the caspase 8-dependent pathway in human colon and prostate cancer cells. Cancer Res 61: 6918-6924, 2001.

17. Yamaguchi K, Lee SH, Eling TE and Baek SJ: Identification of nonsteroidal anti-inflammatory drug-activated gene (NAG-1) as a novel downstream target of phosphatidyl inositol 3-kinase/AKT/GSK-3beta pathway. J Biol Chem 279: 49617-49623, 2004.

18. Liu T, Bauskin AR, Zaunders J, Brown DA, Pankhurst S, Russell PJ and Breit SN: Macrophage inhibitory cytokine 1 reduces cell adhesion and induces apoptosis in prostate cancer cells. Cancer Res 63: 5034-5040, 2003.

19. Macchione E, Epifano O, Stefanini M, Belin D and Canipari R: Urokinase redistribution from the secreted to the cell-bound fraction in granulosa cells of rat preovulatory follicles. Biol Reprod 62: 895-903, 2000. 